\title{
Iron overload syndromes and the liver
}

\author{
Kenneth P Batts \\ Pathology Lab, Division of Gastrointestinal Pathology, Minnesota Gastroenterology, Abbott Northwestern \\ Hospital, Minneapolis, MN, USA
}

\begin{abstract}
Iron can accumulate in the liver in a variety of conditions, including congenital, systemic iron-loading conditions (hereditary hemochromatosis), conditions associated with systemic macrophage iron accumulation (transfusions, hemolytic conditions, anemia of chronic disease, etc), in some hepatitidies (hepatitis C, alcoholic liver disease, porphyria cutanea tarda), and liver-specific iron accumulation of uncertain pathogenesis in cirrhosis. The anatomic pathologist will be faced with the task of determining whether iron accumulation in the liver is significant and, if so, the nature of the disease that lead to the accumulation (ie diagnosis). The tools available to the pathologist include (most importantly) histologic examination with iron stain, quantitative iron analysis, clinical history, laboratory iron tests (serum iron and iron-binding capacity, serum ferritin) and germline genetic analysis for mutations in genes known to be associated with hemochromatosis (HFE, ferroportin, hepcidin, hemojuvelin, transferrin receptor-2). This article provides an overview of the above. Modern Pathology (2007) 20, S31-S39. doi:10.1038/modpathol.3800715
\end{abstract}

Keywords: liver; iron; hemochromatosis; review

\section{Overview and definitions}

Iron can accumulate in the liver in a wide variety of conditions (Table 1), the clinically most important of which is hereditary hemochromatosis $(\mathrm{HH})$. For the anatomic pathologist the distinction between iron accumulation in $\mathrm{HH}$ and other causes can be complicated and success often depends on a combination of morphologic observations, clinical information, genetic studies, and/or quantitative analysis of iron in liver samples. The mere presence of granular iron in liver is referred to a 'hemosiderosis'-this represents a morphologic observation rather than a specific disease entity. Hemochromatosis is a disease state characterized by hemosiderin deposition, however, it has become clear that both adult and juvenile forms of hemochromatosis exist and germline mutations of at least four different genes have been implicated (Table 2).

Our recently expanded understanding of the genetics of hemochromatosis and the variable phenotypic penetration of the genes has made defining 'hemochromatosis' problematic as there is lack of consensus on whether a genotypic or phenotypic definition should be used. Traditionally, 'hemochromatosis' had been defined clinically,

Correspondence: Dr KP Batts, MD, Pathology Lab, Division of Gastrointestinal Pathology, Minnesota Gastroenterology, Abbott Northwestern Hospital, 800 E 28th Street, Minneapolis, MN 55407-373100, USA.

E-mail: kenneth.batts@allina.com

Received and accepted 7 September 2006 based on the presence of a combination of an otherwise unexplained iron overload, frequent presence of iron overload in relatives, and in later stages end-organ damage (liver, pancreas, etc). Before the landmark discovery by Feder et $a l^{1}$ of the HFE gene in 1996, within the medical literature there was an inappropriate use of the terms 'homozygous' and 'heterozygous' hereditary hemochromatosis based on phenotypic findings-we now know that many 'heterozygotes' were in fact 'homozygotes' with low phenotypic penetrance of the gene. Following the discovery of the major gene mutations described below, a 'genetic' definition evolved: '... hemochromatosis is now defined as a disorder of iron metabolism that is inherited as an autosomal recessive trait due to two mutant HFE alleles'. ${ }^{2}$ As data regarding HFE mutation penetrance and the genotypic characterization of non-HFE forms of $\mathrm{HH}$ have become available it appears that neither a purely phenotypic nor genotypic definition is optimal-see penetrance discussion, below.

\section{Iron homeostasis}

The purpose of this article is to provide a practical review of hepatic iron overload for the practicing pathologist. An understanding of the various disease states begins with an understanding of basic iron physiology. Humans modify iron levels through varying the rate of absorption, which occurs primarily in the duodenum, and are incapable of intentionally modifying excretion as there is no normal 
Table 1 Major categories of liver iron overload

Hereditary hemochromatosis
HFE-related (Type 1 hereditary hemochromatosis)
Non-HFE-related (Types $2 \mathrm{~A}, 2 \mathrm{~B}, 3$, and 4 hereditary
hemochromatosis)
Hemosiderosis secondary to systemic disease
Transfusions
Erythrocyte disorders (hemolysis, ineffective erythropoiesis)
Anemia of chronic disease
Cirrhosis-related hemosiderosis
Miscellaneous hemosiderosis
Neonatal hemochromatosis
Hemosiderosis associated with chronic viral or fatty liver
disease
Dietary iron overload

Table 2 Major categories of hereditary hemochromatosis

\begin{tabular}{llll}
\hline & & Mutated gene & Genotypes \\
\hline Type 1 & Adult & HFE & C282Y/C282Y \\
& & & C282Y/H63D \\
Type 2A & Juvenile & Hemojuvelin & \\
Type 2B & Juvenile & Hepcidin & \\
Type 3 & Adult & Transferrin receptor-2 & \\
Type 4 & Adult & Ferroportin & \\
\hline
\end{tabular}

iron excretory mechanism to control-iron loss occurs normally through sloughing of intestinal lining cells and skin cells in addition to menstrual blood loss in women. Recent detailed reviews of this process exist. ${ }^{3}$ Typically iron absorption occurs at a low rate (1-2 mg/day) but this can be upregulated in response to iron deficiency. Key elements of control of iron homeostasis are divalent metal transporter 1 (DMT1), ferroportin, hepcidin, transferrin receptor2(TFR2), and the HFE protein-mutations in all of these except DMT1 have been shown to figure prominently in the various types of $\mathrm{HH}$.

The function of DMT1 is to act as the mediator for iron absorption on the luminal aspect of the mature enterocyte located on duodenal villi (Figure 1-step 1). The expression of DMT1 in the mature, villous duodenal enterocyte is determined by the iron content of the immature duodenal crypt cell-low intracellular iron results in increased DMT1 expression and vice versa. Currently, a disease state related to germline mutations of genes coding for DMT1 is not known.

Ferroportin-1 is an iron transporter located on cells that normally export iron: duodenal crypt enterocytes, macrophages, placental cells, and hepatocytes. ${ }^{4}$ In enterocytes, ferroportin-1, also known by its gene name $S L C 40 A 1$, is located on the basal aspect of the mature duodenal enterocyte (Figure 1-step 2). Ferroportin expression parallels that of DMT1 and is determined by the iron content of the immature duodenal crypt cell-low intracellular iron results in

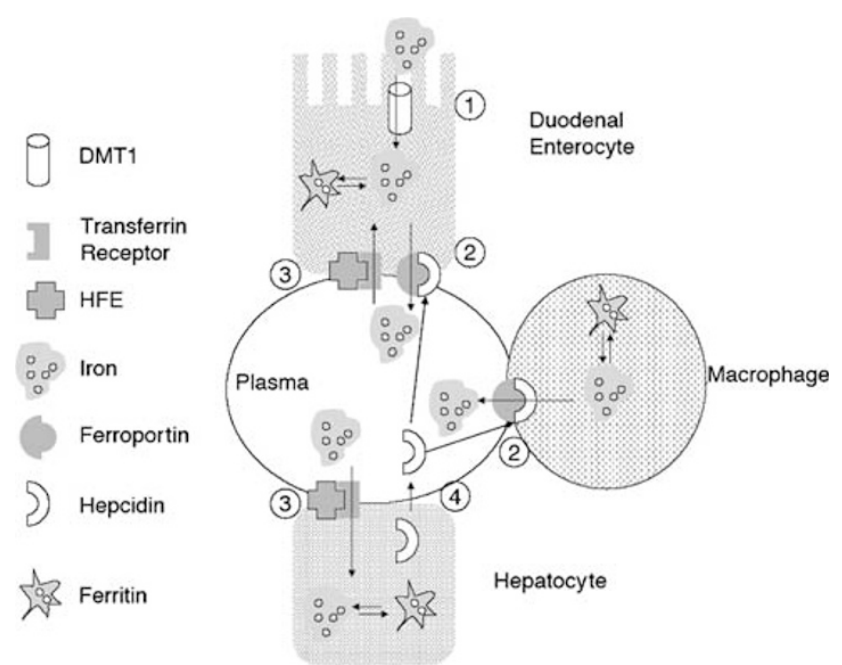

Figure 1 Iron homeostasis. Step 1 represents DMT1-mediated iron absorption into mature enterocytes. Step 2 indicates ferroportin-mediated movement of iron from enterocytes (and macrophages) into the circulation. Step 3 illustrates movement of iron from the circulation into hepatocytes and duodenal enterocytes fostered by the complex of Transferrin receptor 2 and the HFE protein. Step 4 indicates hepatocyte production and excretion of hepcidin into the circulation. Hepcidin downregulates activity of ferroportin, predominantly in duodenal enterocytes and macrophages (step 2).

increased ferroportin-1 expression and vice versa. The function of ferroportin-1 is modified by circulating hepcidin (see below). Mutations in the ferroportin-1 gene characterize 'Type 4' $\mathrm{HH}$ and result in predominant accumulation of iron in macrophages, mimicking secondary iron overload or anemia of chronic disease.

Hepcidin is a plasma peptide produced in hepatocytes (Figure 1-step 4) that appears homeostatically regulated largely by anemia and hypoxemia, likely via transferrin receptor- 2 and the HFE protein (see below) although the precise mechanism and role of iron is unclear. ${ }^{5}$ Hepcidin functions to downregulate ferroportin in enterocytes and macrophages $^{5}$ (Figure 1-step 2). Thus, decreased hepcidin production results in increased movement of iron from intestinal enterocytes (via ferroportin) into plasma and an increase in total body iron content, and vice versa. Hepcidin also will be increased in response to inflammation. It is normally thought to play a role in microbial defense as iron is vital to invading pathogens; by limiting ferroportinmediated release of iron from cells, hepcidin creates a relatively iron deficient milieu which is unfavorable for the pathogen. ${ }^{6}$ If prolonged low hepcidin levels exist, consequences for the patient are accumulation of iron within enterocytes and macrophages and reduced iron availability for erythropoiesis (anemia of chronic disease). Mutations in the hepcidin gene characterize 'Type 2B' $\mathrm{HH}$, a form of juvenile hemochromatosis.

Hepcidin production by hepatocytes is controlled by transferrin receptor-2 (Tfr2), a cell membrane 
protein, and the HFE protein-the complex of Tfr-2 and HFE results in an increase in hepcidin production and corresponding decrease in ferroportinmediated iron transport from enterocytes into plasma (Figure 1-step 3). Tfr-2 and HFE are also felt to be present in duodenal enterocytes. Mutations in the Tfr-2 gene characterize Type $3 \mathrm{HH}$ and mutations in the HFE gene characterize Type $1 \mathrm{HH}$. The latter is the most common form of hereditary iron overload and will be emphasized herein.

\section{Adult ('type 1') hereditary hemochromatosis}

\section{A Brief Medical History of Hemochromatosis}

In 1889, von Recklinghausen identified excess tissue iron obtained at autopsy and named the condition 'hemochromatose'. ${ }^{7}$ This iron was felt to be derived from the bloodstream until 1935 when the British gerontologist Sheldon published his findings from 311 selected patients, concluding that the iron overload was secondary to increased iron absorption. ${ }^{8}$

It was not until 1952 that a therapy became available, when Davis and Arrowsmith reported benefits from treating these patients with phlebotomy. ${ }^{9}$ In 1962, the antemortem diagnosis of hemochromatosis became available with the developing of a grading system for iron in liver samples was developed by Scheuer et al. ${ }^{10}$ Antemortem diagnosis was made easier by the development of reliable serum iron, iron binding capacity, and ferritin testing in the 1960s and 1970s. An HLA locus association was identified by Simon et $a l^{11}$ in 1975 , but the key genetic discovery was the identification of two missense mutations on chromosome $6 \mathrm{p}$ in 1996 by Feder et $a l^{1}$ that accounted for the vast majority of cases of hemochromatosis.

\section{Pathophysiology and Clinical Manifestations of Hemochromatosis}

Type $1 \mathrm{HH}$ is by far the most thoroughly studied form of $\mathrm{HH}$ and is the type associated with mutations in the HFE gene. ${ }^{1}$ It is clear that the fundamental abnormality present in this condition is excessive absorption of iron in the duodenum. Two different theories regarding how the abnormal HFE protein coded by the HFE mutation results in excessive iron accumulation have been proposed (Figure 1-steps 3); they are not mutually exclusive. ${ }^{12}$ The 'hepcidin hypothesis' suggests that the abnormal HFE protein, when complexing with transferrin receptor (Tfr) on the surface of hepatocytes, results in abnormally low hepatocellular and plasma levels of hepcidin being produced. Hepcidin acts as a negative regulator of dietary iron absorption; thus, the HFE mutation results in excessive iron absorption. The 'duodenal crypt cell program- ming hypothesis' proposes that the HFE protein, when complexing with Tfr on the surface of crypt enterocytes, normally serves to facilitate uptake of iron into the crypt enterocyte. An abnormal HFE protein thus fails to facilitate iron uptake into the enterocyte, resulting in a relatively iron deficient cell that will then attempt to excessively absorb iron to correct this perceived deficit.

Regardless of the precise mechanism in adult Type $1 \mathrm{HH}$, the result is a progressive increase of total body iron, reflected by increases in serum transferrin saturation (Tsat) and ferritin, and resulting in abnormal iron deposition, most prominently in hepatocytes but also affecting other organs. In Type $1 \mathrm{HH}$ this iron accumulation is usually clinically inapparent until the fifth or sixth decade, at which time a variety of manifestations may occur (see penetrance discussion below). The most-feared complication, occurring rarely, is progressive hepatic fibrosis and ultimately cirrhosis, accompanied by an increased risk of hepatocellular carcinoma. More common symptoms include diffuse arthropathy and generalized fatigue. Heart disease and heart failure occur in a minority of Type $1 \mathrm{HH}$ patients but are dominant features in the rare juvenile forms of $\mathrm{HH} .{ }^{13}$ A variety of endocrine disorders are also seendiabetes mellitus, hypogonadism, and hypothyroidism. Rarely seen now is cutaneous pigmentation ('bronze diabetes'). Less well characterized are potential increased risks of extrahepatic malignancy and for infections with systemic iron overload.

\section{Genetics of adult ('type 1') hereditary hemochromatosis}

\section{Population Genetics}

It appears likely that the initial hemochromatosis mutation occurred within the last 100-120 generations (at 2800) years or so in Northwestern Europe in a Celtic, Viking, or Germanic population. ${ }^{14}$ There has been speculation that the propagation of this gene could have occurred because the adverse complications of this mutation did not become evident until after the reproductive years and the iron stores were of benefit to a younger adult population living in a region of possibly limited dietary intake of iron. ${ }^{14}$ Proof of this hypothesis remains lacking, however. The worldwide distribution of hemochromatosis reflects the migrations of the originally affected populations-Caucasians in Northwestern Europe, Canada, USA, Australia, New Zealand, and South Africa all have roughly similar gene frequencies of 0.06-1.0 and heterozygous carriage in $10-15 \%$ for the major gene mutations.

\section{The HFE Gene}

In 1996 Feder et al ${ }^{1}$ identified two mutations in an MHC class I gene at chromosome 6p21 which is now 
named the 'HFE' gene and defines Type $1 \mathrm{HH}$. The 'major' mutation was a $G$ to A substitution at nucleotide 845 which lead to a cysteine to tyrosine substitution at the amino acid 282, now commonly referred to a the 'C282Y' mutation. The 'minor' mutation was a $\mathrm{C}$ to $\mathrm{G}$ change at nucleotide 187 which resulted in a histidine to aspartic acid substitution at position 63 ('H63D'). Among patients with marked iron overload ('phenotypic hemochromatosis'), HFE mutations dominate (Type $1 \mathrm{HH}$ ). Approximately $80-85 \%$ of these patients (range 59-100\%) will demonstrate a C282Y/C282Y genotype and approximately 5\% (range $4-8 \%$ ) a $\mathrm{C} 282 \mathrm{Y} /$ H63D phenotype. ${ }^{13}$ Rare HFE mutations in loci other than amino acids 282 or 63 do occur. ${ }^{12,15}$ The C282Y/wild-type, H63D/wild-type, and H63D/ $\mathrm{H}_{63 \mathrm{D}^{16}}$ genotypes are not associated with phenotypic disease although minor elevations in iron saturation may be present.

\section{HFE Protein}

The HFE gene codes for a 343 amino acid protein that is similar to class $1 \mathrm{MHC}$ proteins. ${ }^{12}$ The $\mathrm{C} 282 \mathrm{Y}$ mutation results in a mutant HFE protein which undergoes accelerated degradation and decreased expression on the cell surface. See the preceding discussion under 'pathogenesis of $\mathrm{HH}$ '.

\section{HFE Mutations and Gene Penetrance}

The discovery of the HFE mutations by Feder et $a l^{1}$ resulted in a dilemma as to whether $\mathrm{HH}$ should be defined by the clinical and laboratory phenotype or by the genotype. Simply using the genotype is problematic as the expression ('penetrance') of the gene varies widely. There appear to be modifier genes which modulate the expression of HFE mutations ${ }^{17,18}$ which, when coupled with the individual variations of iron loss (such as in menstruating women), result in considerable variation in the clinical expression of at risk (C282Y/C282Y and C282Y/H63D) genotypes.

A system for grading the degree of phenotypic expression of iron overload was developed by Brissot et $a l^{19}$ using a 0-4 scale. In this system, asymptomatic individuals fall into grades $0-2$ : grade 0 shows no clinical or serum iron test abnormalities, grade 1 increased serum TSat with normal ferritin, and grade 2 has increased TSat plus increased serum ferritin. Grade 3 encompasses patients with minor symptoms minimally impacting quality of life and grade 4 those with potentially lethal symptoms.

Among $282 \mathrm{Y}$ homozygous individuals, the effect of gender on penetrance is particularly evident in grades 1 and 2 . In a review, which compiled 153 C282Y homozygous males, $73.7 \%$ (range $60-86 \%$ ) demonstrated an elevated TSat (at least grade 1 disease), with a mean value of $65.3 \% .{ }^{20}$ In contrast, among 206 women, $50.6 \%$ had an elevated Tsat (range $40-100 \%$ ) with a mean value of $47.1 \% .^{20}$ Elevated serum ferritin levels (at least grade 2 disease) is seen less commonly than grade 1 both in men $(65.4 \%$, range $34-100 \%)$ and women $(40.5 \%$, range $9-63 \%) .{ }^{20}$

Among C282Y homozygotes with minor symptoms (at least grade 3 disease), joint pain (36\% of all patients) and fatigue (32\% of all patients) are most common with dark skin, diabetes, and elevations in serum aminoaspartate (AST) being present in about $6-7 \%$ of patients each. ${ }^{20}$ Grade 4 disease (potentially lethal disease) appears to be very uncommon in C282Y homozygotes, Beutler et $a l^{21}$ estimating an occurrence of only about $1 \%$.

\section{Non-HFE Hemochromatosis in Adults}

Among the remaining $5-10 \%$ of phenotypic $\mathrm{HH}$ cases in adults, two major categories have emerged. Mutations in transferrin receptor 2 (Tfr 2) result in a disease phenotypically similar to Type $1 \mathrm{HH}$; this has been referred to as 'Type 3' $\mathrm{HH}^{12}$ The ferroportin-1 gene (SCL40A1) on chromosome 2q32 is mutated in 'Type 4' $\mathrm{HH}^{12}$ In contrast to the aforementioned types of $\mathrm{HH}$, iron accumulation may occur predominantly in reticuloendothelial cells and phlebotomies are not beneficial. ${ }^{17}$ A recent detailed review exists. ${ }^{22}$

\section{Juvenile hemochromatosis}

Juvenile forms of hereditary hemochromatosis are much less common than adult forms and come in two forms, referred to as 'Type 2A' and 'Type 2B' HH. ${ }^{17}$ Type $2 \mathrm{~A}$ reflects a mutation in the hemojuvelin gene at chromosome 1a21, is autosomal recessive, and tends to present with prominent cardiac iron deposition before the age of 30 with resulting risk of heart failure and potential hypogonadism. Hemojuvelin appears to function as a regulator of hepcidin. ${ }^{23}$ Type $2 \mathrm{~B}$ is the result of a mutation in the hepcidin gene at 19q13, is also autosomal recessive, and phenotypically is similar to HFE-type HH. ${ }^{17}$

\section{Neonatal Hemochromatosis}

Neonatal hemochromatosis is not inherited, is unrelated to juvenile and adult forms of $\mathrm{HH}$, and remains of uncertain pathogenesis. ${ }^{24}$ Neonatal hemochromatosis may present in late second or third trimester as fetal loss or in the hours to weeks after birth as acute liver failure and often multiorgan failure. Histologically, iron deposition within hepatocytes rather than Kupffer cells is typical, with iron deposition to a lesser degree occurring in non-hepatic organs as well. In addition to the iron deposition, hepatic injury in the form of occasionally marked lobular necrosis and regeneration occurs typically and cirrhosis is common. ${ }^{24}$ 


\section{Histopathology of adult hereditary hemochromatosis}

The hallmark of Types 1 (and 3) HH is the deposition of hemosiderin in hepatocytes and biliary epithelium (Figures 2-4) rather than reticuloendothelial cells. ${ }^{25,26}$ Hemosiderin is insoluble and particulate in nature and will appear granular with iron stains. ${ }^{26}$ Alternatively, iron may be deposited in the form of ferritin which is soluble and characterized by diffuse, non-granular light blue staining of the hepatocyte or macrophage cytoplasm and is very non-specific and not characteristic of genetic hemochromatosis.

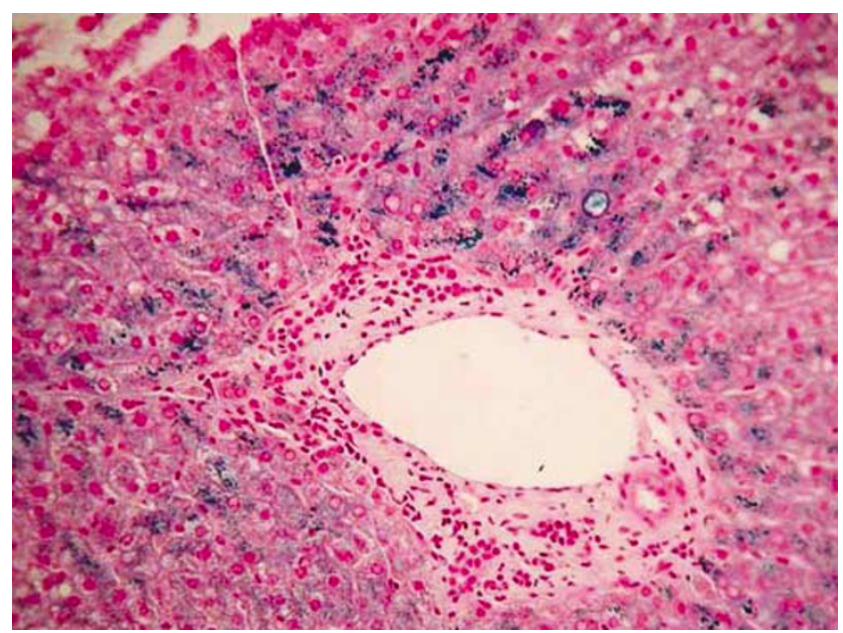

Figure 2 Hemosiderin in Zone 1 hepatocytes in Type $1 \mathrm{HH}$. In HFE-related Type $1 \mathrm{HH}$, iron accumulation begins as hemosiderin in periportal ('zone 1') hepatocytes. The clinical significance will be related to rate of iron accumulation. The calculated iron content for this biopsy was $7633 \mu \mathrm{g} / \mathrm{g}$ dry weight liver and the patient's age was 24 , resulting in a calculated hepatic iron index of 5.7 (see also Figure 7). At this rate of accumulation, fibroticrange iron accumulation (approximately 15000 or greater) will occur eventually. In contrast, if the patient had been 84 years old, the hepatic iron index would have been 1.63 , reflecting relatively mild gene penetrance and little risk of significant iron-related symptoms.

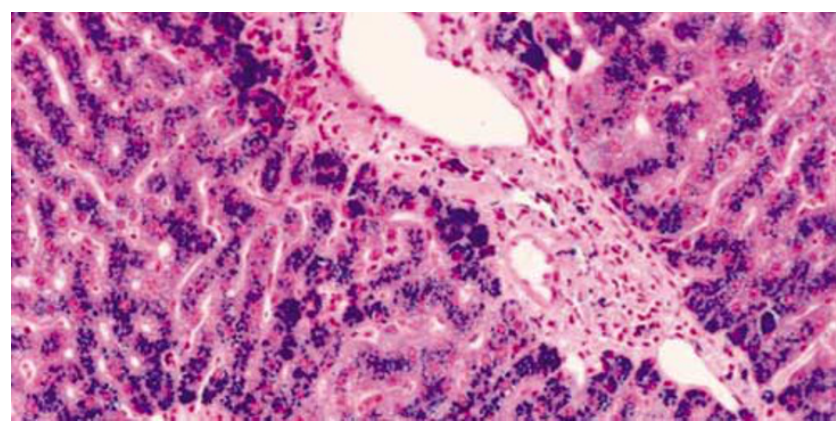

Figure 3 Panlobular hemosiderin accumulation in Type $1 \mathrm{HH}$. In this case, the entire lobule has accumulated iron ('grade 4') and the measured iron content was $23000 \mu \mathrm{g} / \mathrm{g}$ dry weight liver. This represents highly penetrant $\mathrm{HH}$ regardless of age, this liver is at high risk of developing significant fibrosis in the near-term, and the likelihood of extrahepatic manifestations of $\mathrm{HH}$ high as well.
In early phases of disease, hemosiderin is deposited in periportal (zone 1) hepatocytes (Figure 2). As progressive iron accumulation takes place, midzonal (zone 2) and centrilobular (zone 3) hepatocytes will progressively accumulate iron (Figure 3 ) as will biliary epithelium (Figure 4). A variety of systems exist for grading hepatic iron deposition histologically, almost all using the general principles of grade increasing with greater amounts of iron and progressive involvement of hepatocytes beginning in zone 1 (periportal) and eventually extending to zone 3 (pericentral). By contrast, iron deposition in hematologic disorders occurs primarily in the reticuloendothelial system (Kupffer cells) and, when present in large quantities, 'spills over' into the hepatocytes to a lesser degree (Figure 5). Thus, the finding of iron deposited primarily in Kupffer cells is not in keeping with hemochromatosis (other than the rare HFE 4 'ferroportin disease').

As iron accumulates, eventually individual hepatocytes will accumulate lethal levels of iron and undergo 'sideronecrosis'. The locally released iron is then taken up into macrophages, however hepatocellular iron will continue to dominate (Figure 4). Macrophage iron is linked to progressive fibrosis. ${ }^{26}$ Precirrhotic portal and periportal fibrosis takes on a 'holly leaf' configuration. When cirrhosis is present, it is bland in nature with fine fibrous tissue septa surrounding regenerative nodules.

Steatosis is not an a priori component of $\mathrm{HH}$, however, with the high prevalence of obesity among populations prone to develop Type $1 \mathrm{HH}$ it is not surprising that fat is not uncommonly present in $\mathrm{HH}$. In Australia, 41\% of C282Y homozygote Type 1 $\mathrm{HH}$ patients had some steatosis and in $14.5 \%$ it was moderate or severe. ${ }^{27}$ Concurrent steatosis may be a co-factor for accelerating fibrosis in $\mathrm{HH} .{ }^{27}$ Paradoxi-

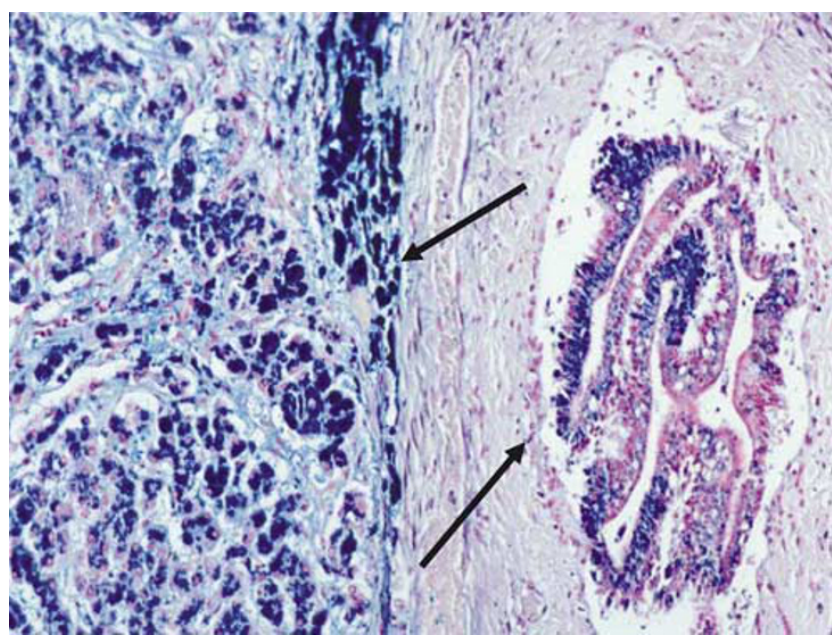

Figure 4 Marked iron accumulation in cirrhosis due to Type 1 HH. Here, in addition to grade 4 iron accumulation in hepatocytes, many iron-laden macrophages are present (upper arrow), a reflection of prior hepatocyte sideronecrosis and redistribution of iron into macrophages. Accumulation of iron in biliary epithelium is not uncommon as well (lower arrow). 


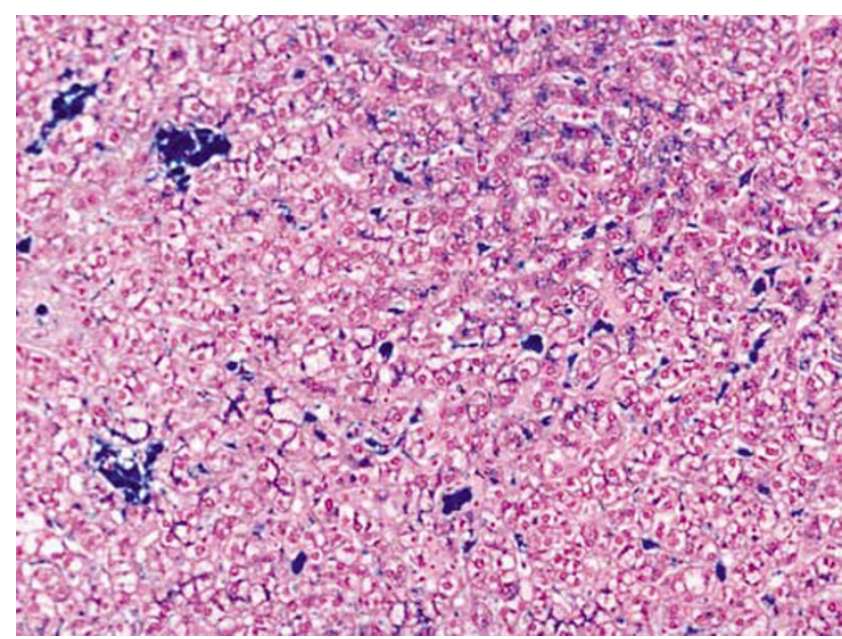

Figure 5 Macrophage (Kupffer cell) hemosiderosis secondary to transfusions. Macrophage-predominant iron accumulation is typical of iron deposition secondary to transfusions, other hematologic disorders, or anemia of chronic disease rather than Type 1 (HFE-related) HH. Ferroportin disease (Type $4 \mathrm{HH}$ ) could show this pattern, however.

cally, however, there is evidence that obesity may suppress the likelihood of C282Y homozygotes demonstrating phenotypic expression of their disease in the form of an elevated serum iron saturation. ${ }^{28}$

Patients with $\mathrm{HH}$ have an estimated 240-fold increased relative risk of developing hepatocellular carcinoma, with the degree of risk correlating with the amount and duration of iron overload and degree of fibrosis. ${ }^{29}$ Currently iron is felt to play a carcinogenic of co-carcinogenic role through the production of iron-mediated oxidative stress, tumor growth facilitation, and/or immune system modification. ${ }^{29}$ Another histologic feature described in the hemochromatotic liver is the iron free focus, (Figure 6) characterized by a localized zone of relative reduction of hepatocytic iron. ${ }^{29}$ This lesion is purported to represent an early step in the development of hepatocellular carcinoma. Both the clinician and the pathologist should maintain a high index of suspicion for hepatocellular carcinoma in $\mathrm{HH}$ as it may occur in up to one-third of patients with cirrhotic stage hemochromatosis. ${ }^{30}$

\section{Differential diagnosis of hemosiderosis}

It should be recognized that hepatic hemosiderin deposition may occur in a wide variety of disease states other than genetic hemochromatosis (Table 1) although mechanisms remain obscure in most cases. In general, thorough clinical evaluation and careful histologic examination can distinguish these conditions from genetic hemochromatosis although biochemical analysis of liver tissue iron (see below) or genotypic analysis may be necessary in ambiguous cases.

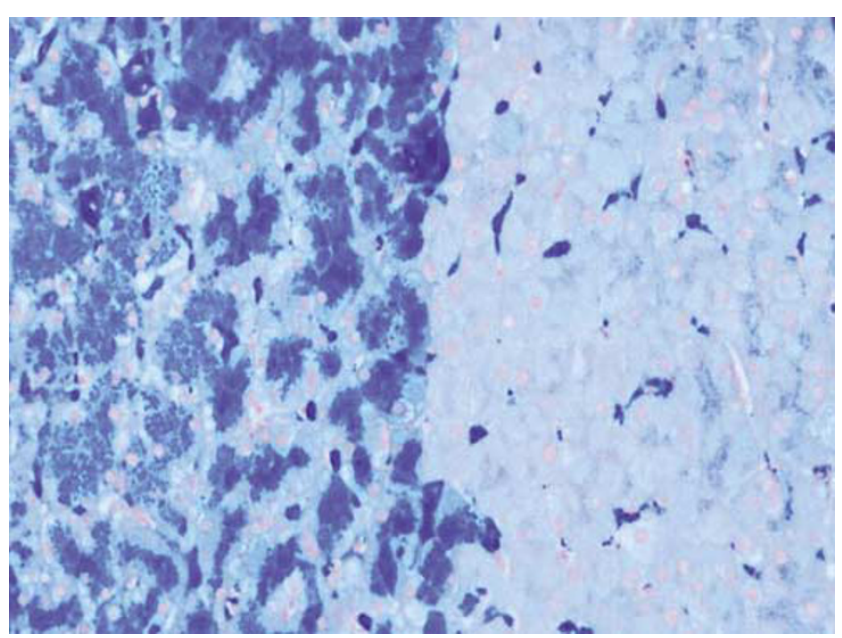

Figure 6 Iron free focus in hereditary hemochromatosis. The hepatocytes on the right side of the field contain almost no iron, in contrast to those on the left side of the field, suggesting the 'iron free' hepatcytes represent a recent-onset clone. Note that Kupffer cells contain some iron within the iron free focus.

\section{Hematologic Disorders}

Transfusions and chronic hemolytic disorders commonly lead to hepatic hemosiderin deposition. Transfusional iron tends to accumulate in Kupffer cells and is thus easily distinguished from the hepatocellular iron of most forms of genetic hemochromatosis. Iron following hemolysis tends to be deposited in both hepatocytes and Kupffer cells and thus demonstration of hemolysis by laboratory means is very helpful in distinguishing hemolysisrelated hemosiderosis from genetic hemochromatosis. Anemia of chronic disease can also lead to Kupffer cell iron deposition and it is not uncommon for very mild Kupffer cell iron to be present with no identifiable clinical correlate.

\section{Cirrhosis-Associated Hemosiderosis}

Accumulation of hemosiderin is fairly common in cirrhosis of any type; Ludwig et $a l^{31}$ noted stainable hemosiderin in 145/449 (32.4\%) of cirrhotic livers. Furthermore, formal iron quantitation revealed increased hepatic iron concentration in 91/449 $(20.3 \%)$, including 38 cases $(8.5 \%)$ in which the level was in the $\mathrm{HH}$ range (hepatic iron index >1.9). ${ }^{26}$ Dominant hepatocellular iron, similar to that seen in $\mathrm{HH}$, is not uncommon; however, in this study it was felt that $\mathrm{HH}$ only accounted for five of 145 instances of hepatic hemosiderosis occurring in cirrhosis. Other authors have noted similar observations. $^{32}$

In cases of cirrhosis with iron deposition, one is usually able to determine whether homozygous $\mathrm{HH}$ is present using traditional means of assessment, however, in ambiguous cases genetic analysis may play a helpful role. The etiology for the iron 
deposition remains unclear, but patients with biliary cirrhosis seem to be less prone to accumulate iron $(7-20 \%)$ than are patients with non-biliary cirrhosis $(22-67 \%)$.

In summary, while some cases of cirrhosis with iron deposition could represent mildly penetrant homozygous $\mathrm{HH}$ or heterozygous $\mathrm{HH}$, it seems clear that iron deposition can occur in cirrhosis as a secondary phenomenon, may be hepatocellulardominant morphologically, and may give 'false positive' hepatic iron indices $>1.9$.

\section{Alcohol \\ Alcoholic steatohepatitis is one of the more common non-hemochromatotic causes of hepatic hemo- siderosis and may reflect increased intestinal iron absorption. ${ }^{33}$ Histological features include fatty infiltration and varied degrees of inflammatory reaction. Generally, stainable hepatic iron is only mild in such instances, but at times it is sufficient to cause confusion with regard to the question of genetic hemochromatosis. Measurement of hepatic iron concentration was originally applied to making this distinction ${ }^{34}$ and may still play a role.}

\section{Chronic Viral Hepatitis}

Chronic viral hepatitis is not uncommonly accompanied by hepatic hemosiderin, which is generally mild and much less than would be expected for reasonably penetrant $\mathrm{HH}$. The hemosiderin deposits can be present in Kupffer cells, hepatocytes (with a zone 1-3 gradient), and/or portal areas. ${ }^{35}$ Depletion of the iron through phlebotomy appears to lower serum transaminase values, decrease inflammation, and may slow progression of the hepatitis C. ${ }^{36}$ While data have been variable, it is not clear that the amount of stainable iron predicts the likelihood of viral eradication with interferon and ribavirin therapy. ${ }^{37}$

\section{Quantitative analysis of liver tissue iron}

\section{Overview}

In the past 25 years, measurement of iron concentration in liver biopsy specimens has proven to be a valuable diagnostic tool that has also served to broaden our concept of hemochromatosis. ${ }^{38}$ Its value has diminished in the era of genetic testing but iron quantitation can still play a diagnostic and prognostic role in some instances. Tissue iron analysis must be performed in a highly qualified laboratory where rigorous control of processing and analytical procedures are followed to assure accurate results. The actual analysis is typically performed in a graphite atomic absorption spectrophotometer. The results are reported in $\mu \mathrm{mol} / \mathrm{g}$ dry weight or in $\mu \mathrm{g} / \mathrm{g}$ dry weight. Normal values in the Mayo Metals
Laboratory are $7.2-39.4 \mu \mathrm{mol}(400-2200 \mu \mathrm{g})$ per gram dry weight in men and 1.8-28.6 $\mu \mathrm{mol}(100-1600 \mu \mathrm{g})$ per gram dry weight in women. The variation of repeated determinations on the same test sample is approximately $5 \%$.

Mild to moderate iron overload (1-2+) generally correlates with quantitative iron values between 3000 and $10000 \mu \mathrm{g} \mathrm{Fe} / \mathrm{g}$ dry weight liver (Figure 2). Grades 3 and 4 iron histologically generally reflect $>10000 \mu \mathrm{g} \mathrm{Fe} / \mathrm{g}$ dry weight liver (Figure 3). In uncomplicated $\mathrm{HH}$, most patients with iron concentrations of $22000 \mu \mathrm{g} \mathrm{Fe} / \mathrm{g}$ dry weight liver likely will have developed fibrosis as well. ${ }^{38}$

The concept of the hepatic iron index (HII) was introduced by Bassett et $a l^{38}$ in 1986, and it has proven useful, especially in the interpretation of mild to moderate degrees of hepatic iron overload. The HII was developed to account for the progressive iron accumulation that occurs over time in $\mathrm{HH}-\mathrm{a}$ given amount of iron at age 25 indicates a more rapid accumulation of iron than the same amount of iron in a 50-year-old individual (Figure 7). The index is simply calculated by dividing the hepatic iron concentration in $\mu$ moles/ $\mathrm{g}$ by the patient's age and is expressed in micromoles/g/year. As the hepatic iron concentration is often reported in $\mu \mathrm{g} / \mathrm{g}$, the value must be divided by 55.8 to convert it to $\mu$ moles/g. An HII of 1.9 or greater (in normals, the index is usually $<1.0$ ) was previously felt to indicate 'homozygous hemochromatosis'. However, in the context of subsequent

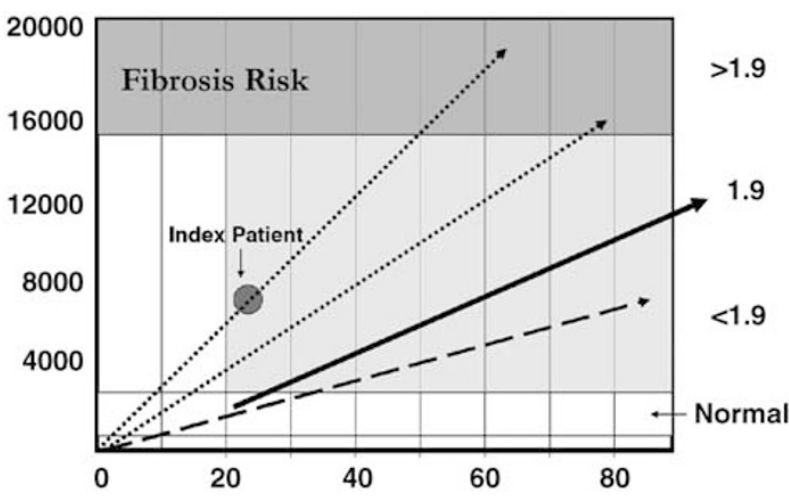

Figure 7 Quantitative iron analysis and hepatic iron index (HII). The quantity of measured iron (left $y$-axis), in the context of the patient's age ( $x$-axis), results in the hepatic iron index, defined by iron quantity in micrograms divided by the patient's age divided by the atomic weight of iron (55.8). An HII value of 1.9 (solid line) represents the traditional (pre-genetic testing) distinction between 'homozygotes' (HII >1.9) and 'heterozygotes (HII <1.9). Current thought is that values $>1.9$ (dotted lines) reflect patients at risk for developing adverse consequences from iron overload and values $<1.9$ (hatched line) patients at little/no risk for adverse consequences from iron overload; many true C282Y homozygotes will have HII values below 1.9. The patient illustrated in Figure 2 is plotted as an example-if one assumes abnormal iron accumulation beginning early in life and continued iron accumulation, this patient will find themself with fibrosiscausing levels of liver iron while still in mid-adulthood if untreated. 
knowledge gleaned about the penetrance of C282Y homozgosity, it appears that an HII of 1.9 or greater actually identifies patients at risk for symptomatic disease (ie clinical grades 3 and 4). Presence of stainable hepatocyte iron with zone 1 predominance and HII values $<1.9$ are likely largely composed of C282Y homozygous with mild penetrance (clinical grades 1 and 2) and C282Y heterozygotes.

\section{Utility of Hepatic Iron Concentration}

There is a reasonable correlation between visualized hemosiderin and quantitative iron values, in contrast to quantitative copper analysis. Specifically, if the pathologist finds little stainable iron, measurement of hepatic iron concentration is unnecessary. Conversely, if there is histological evidence of heavy iron deposition, the hepatic iron concentration may be superfluous. For diagnostic purposes, quantitative iron analysis adds little to histologic estimation of iron content in the context of biochemical, clinical, and genetic information. However, quantitative iron analysis can provide objective data on disease penetrance and estimated phlebotomy duration before iron depletion is achieved.

Quantitative iron analysis has been applied to the potentially difficult differential diagnosis of alcoholic siderosis vs hereditary hemochromatosis. Most literature on this topic was published before the discovery of the HFE gene, however, quantitative analysis still may occasionally play a role. In alcoholic liver disease, the hepatic iron concentration rarely exceeds two to three times the upper limit of normal, ${ }^{39}$ and the HII will generally be $<1.9$. On the other hand, alcoholic patients with heavy iron overload (for example, $>5$ times normal hepatic iron concentration) have been shown to have genetic hemochromatosis of the homozygous form. ${ }^{40}$ Alcohol likely works in synergy with iron to aggravate tissue injury and may give rise to cirrhosis in the hemochromatotic earlier than would be expected with iron overload alone. ${ }^{40,41}$

Although the validity of the hepatic iron index has been confirmed in several studies, there are instances where available evidence and clinical judgment support another interpretation. Firstly, iron quantitation and hepatic iron index determination for diagnostic purposes should be restricted to cases in which the histologic distribution of suggests genetic hemochromatosis, that is hepatocellular iron predominates over Kupffer cell iron, and a reasonable clinical explanation for iron overload does not exist. Transfusional hemosiderosis, particularly in younger individuals, can easily lead to hepatic iron indices in excess of 1.9. Second, the utility of the hepatic iron index in the pediatric population has not been demonstrated and thus should be used in this group with utmost caution if at all. Third, as discussed above, it should be kept in mind that the index was originally applied to cases of early hemochromatosis and must be interpreted cautiously in patients with advanced chronic liver disease as 'false positive' hepatic iron indices in cirrhosis are common. ${ }^{31}$

As measurement of hepatic iron concentration is not always available, Deugnier et $a l^{42}$ have investigated the utility of a histologic hepatic iron index based on detailed and systematic grading of iron deposits in various locations of the liver lobule. In their hands, this provided similar results to biochemically determined HII. A computerized assessment of hepatic iron can be performed on a standard histologic slide stained for iron as well. ${ }^{43}$

\section{References}

1 Feder JN, Gnirke A, Thomas W, et al. A novel MHC class-1-like gene is mutated in patients with hereditary hemochromatosis. Nat Genet 1996;14:399-408.

2 Edwards CQ, Ajioka RS, Kushner JP. Hemochromatosis: a genetic definition. In: Barton JC, Edwards CQ (eds). Hemochromatosis. Genetics, Pathophysiology, Diagnosis, and Treatment. Cambridge University Press: Cambridge, 2000, pp 8-11.

3 Yen AW, Fancher TI, Bowlus CL. Revisiting hereditary hemochromatosis: current concepts and progress. Am J Med 2006;119:391-399.

4 Wessling-Resnic M. Iron Imports III. Transfer of iron from the mucosa into circulation. Am J Physiol Gastrointest Liver Physiol 2006;290:G1-G6.

5 Ganz T, Nemeth E. Iron Imports IV. Hepcidin and regulation of body iron metabolism. Am J Physiol Gastrointestinal Liver Physiol 2006;290:G199-G203.

6 Pietrangelo A, Trautwein C. Mechanisms of disease: the role of hepcidin in iron homeostasis-implications for hemochromatosis and other disorders. Nat Clin Prac Gastroenterol Hepatol 2004;1:39-45.

7 von Recklinghausen FD. Uber hemochromatose. Tagelb Versamml Natur Artze Heidelberg 1889;62:324-325.

8 Sheldon JH. Haemochromatosis. Oxford University Press: London, 1935.

9 Davis WD, Arrowsmith WR. The effect of repeated phlebotomies in hemochromatosis; report of three cases. J Lab Clin Med 1952;39:526-532.

10 Scheuer PJ, Williams R, Muir AR. Hepatic pathology in relatives of patients with hemochromatosis. J Pathol Bacteriol 1962;84:53-64.

11 Simon M, Pawlotsky Y, Bourel M, et al. Hemochromatose idiopathique: maladie associee a l'antigene tissulaire HLA 3. Nouv Presse Med 1975;19:1432.

12 Fleming RE, Britton RS, Waheed A, et al. Pathogenesis of hereditary hemochromatosis. Clin Liver Dis 2004;8: 755-773.

13 Roetto A, Camaschella C. New insights into iron homeostasis thought the study of non-HFE hereditary haemochromatosis. Best Practice Res Clin Haematol 2005;18:235-250.

14 Fairbanks VF. Population genetics. In: Barton JC, Edwards CQ (eds). Hemochromatosis. Genetics, Pathophysiology, Diagnosis, and Treatment. Cambridge University Press: Cambridge, 2000, pp 42-50.

15 Pointon JJ, Wallace D, Merryweather-Clarke AT, et al. Uncommon mutations and polymorphisms in the hemochromatosis gene. Genetic Test 2000;4:151-161. 
16 Gochee PA, Powell LW, Clullen DJ, et al. A populationbased study of the biochemical and clinical expression of the H63D hemochromatosis mutation. Gastroenterology 2002;122:646-651.

17 Aquilar-Martinez P, Schved JF, Brissot P. The evaluation of hyperferritinemia: an updated strategy based on advances in detecting genetic abnormalities. Am J Gastroenterol 2005;100:1185-1194.

18 Levy JE, Montross LK, Andrews NC. Genes that modify the hemochromatosis phenotype in mice. J Clin Invest 2000;105:1209-1216.

19 Brissot P, Troadec MB, Loreal O. The clinical relevance of new insights in iron transport and metabolism. Curr Haematol Rep 2004;3:107-115.

20 Waalen J, Nordestgaard BG, Beutler E. The penetrance of hereditary hemochromatosis. Best Practice Res 2005; 18:203-220.

21 Beutler E, Felitti VJ, Koziol JA, et al. Penetrance of 845G-A (282Y) HFE hereditary haemochromatosis mutation in the USA. Lancet 2002;359:211-218.

22 DeDomenico I, Ward DM, Musci G, et al. Iron overload due to mutations in ferroportin. Haemoatologica 2006; 91:92-95.

23 Celec P. Hemojuvelin: a supposed role in iron metabolism one year after its discovery. J Mol Med 2005;83:521-525.

24 Whitington PF. Fetal and infantile hemochromatosis. Hepatology 2006;43:654-660.

25 Brunt EM, Olynyk JK, Britton RS, et al. Histological evaluation of iron in liver biopsies: relationship to HFE mutations. Am J Gastroenterol 2000;95:1788-1893.

26 Deugnier YM, Loreal O, Turlin B, et al. Liver pathology in genetic hemochromatosis: a review of 135 homozygous cases and their biochemical correlations. Gastroenterology 1992;102:2050-2059.

27 Powell E, Ali A, Clouston AD, et al. Steatosis is a cofactor in liver injury in hemochromatosis. Am J Gastroenterol 2005;129:1937-1943.

28 Laine F, Jouannolle A-M, Morcet J, et al. Phenotypic expression in detected $\mathrm{C} 282 \mathrm{Y}$ homozygous women depends on body mass index. J Hepatol 2005;43: 1055-1059.

29 Deugnier YM, Guyader D, Crantock L, et al. Primary liver cancer in genetic hemochromatosis: a clinical, pathogenetic study of 54 cases. Gastroenterology 1993; 104:228-234.

30 Niederau C, Fischer R, Purschel A, et al. Long-term survival in patients with hereditary hemochromatosis. Gastroenterology 1996;110:1107-1119.
31 Ludwig JL, Hashimoto E, Porayko MK, et al. Hemosiderosis in cirrhosis: a study of 447 native livers. Gastroenterology 1997;112:882-888.

32 Fiel MI, Schiano TD, Bodenheimer HC, et al. Hereditary hemochromatosis in liver transplantation. Liver Transplant Surg 1999;5:50-56.

33 Duane P, Raja KB, Simpson RJ, et al. Intestinal iron absorption in chronic alcoholics. Alcohol Alcoholism 1992;27:539-544.

34 Powell LW, Fletcher LM, Halliday JW. Distinction between haemochromatosis and alcoholic siderosis. In: Hall P (ed). Alcoholic Liver Disease, 2nd edn. Edward Arnold: London, 1995, pp 199-216.

35 Fabris C, Toniutto P, Scott CA, et al. Serum iron indices as a measure of iron deposits in chronic hepatitis D. Clin Chim Acta 2001;304: 49-55.

36 Yano M, Hayashi $\mathrm{H}$, Waksawa S, et al. Long term effects of phlebotomy on biochemical and histological parameters of chronic hepatitis C. Am J Gastroenterol 2002;97:133-137.

37 Hofer H, Osterreicher C, Jessner W, et al. Hepatic iron concentration does not predict response to standard and pgylated-IFN/ribavirin therapy in patients with chronic hepatitis C. J Hepatol 2004;40: 1018-1022.

38 Bassett ML, Halliday JW, Powell LW. Value of hepatic iron measurements in early hemochromatosis and determination of the critical iron level associated with fibrosis. Hepatology 1986;6:24-29.

39 LeSage GD, Baldus WP, Fairbanks VF, et al. Hemochromatosis: genetic or alcohol-induced? Gastroenterology 1983;84:1471-1477.

40 Adams PC, Agnew S. Alcoholism in hereditary hemochromatosis revisited; prevalence and clinical consequences among homozygous siblings. Hepatology 1996;23:724-727.

41 Irving MG, Halliday JW, Powell LW. Association between alcoholism and increased hepatic iron stores. Alcohol: Clin Exp Res 1988;12:7-13.

42 Deugnier YM, Turlin B, Powell LW, et al. Differentiation between heterozygotes and homozygotes in genetic hemochromatosis by means of a histological hepatic iron index: a study of 192 cases. Hepatology 1993;19:30-34.

43 Olynyk J, Hall P, Sallie R, et al. Computerized measurement of iron in liver biopsies: a comparision with biochemical iron measurement. Hepatology 1990; 12:26-30. 